\title{
Entanglement in a Quantum Mixed-Spin Chain
}

\author{
Xiang Hao and Shiqun Zhu* \\ China Center of Advanced Science and Technology(World Laboratory), \\ P. O. Box 8730, Beijing 100080, People's Republic of China \\ and \\ School of Physical Science and Technology, Suzhou University, \\ Suzhou, Jiangsu 215006, People's Republic of China**
}

\begin{abstract}
The entanglement in a general mixed spin chain with arbitrary spin $S$ and $1 / 2$ is investigated in the thermodynamical limit. The entanglement is witnessed by the magnetic susceptibility which decides a characteristic temperature for an entangled thermal state. The characteristic temperature is nearly proportional to the interaction $J$ and the mixed spin $S$. The bound of negativity is obtained on the basis of the magnetic susceptibility. It is found that the macroscopic magnetic properties are affected by the quantum entanglement in the real solids. Meanwhile, the entanglement can be quantitatively evaluated by the thermodynamical observable.
\end{abstract}

PACS Number: 03.67.Mn, 03.65.Ud, 75.10.Jm

* Corresponding author, email: szhu@suda.edu.cn

** Mailing address 
The quantum entanglement can exhibit the quantum non-locality that cannot be explained by the classic laws. The entanglement is very essential in the quantum computation and quantum information processing [1,2]. As a crucial resource, the entanglement has been investigated by some basic measures [3-5] in many systems [69]. The entanglement of formation [3] and the relative entropy of entanglement [4] are used for two qubits. The negativity [5] is used to qualify the entanglement for any bipartite system.

The entanglement consists not only in microscopic quantum systems [6,7], but also in macroscopic systems [8-10]. It is demonstrated that the macroscopic thermodynamical observable contains the entanglement. From the separability criterion [11], the entanglement witness [12-19] has been proposed to evaluate the entanglement in the real solids. An entanglement witness is an observable which has an positive expected value for a separable (unentangled) state, a negative value for an entangled state. The effect of the entanglement in solids has been observed in the experimental situation [10]. The thermal energy [13-16], the heat capacity [19], and the magnetic susceptibility [10, 20-22] have been considered as the witness for the quantum spin- $\frac{1}{2}$ systems. They decide a characteristic temperature below which an entangled thermal state is obtained. Besides the general spin- $\frac{1}{2}$ system [23], the properties of the quantum mixed or alternating spin system $[24,25]$ is also attracting due to the peculiar phenomenon of spin-Peierls transition. For an inorganic compound, the quasi one-dimensional bimetallic chains $\mathrm{ACu}(\mathrm{pbaOH})\left(\mathrm{H}_{2} \mathrm{O}_{3}\right) \cdot 2 \mathrm{H}_{2} \mathrm{O}$, with each unit cell containing two different spins $(S, 1 / 2)$, have been synthesized [25]. The compounds of ACu may be considered as a Heisenberg mixed spin $(S, 1 / 2)$ chain with antiferromagnetic interactions. It is necessary to study the entanglement in the real mixed spin systems.

In this Letter, the entanglement in a general mixed spin chain is investigated on the basis of the experimental measurements of the magnetic susceptibility. The entanglement is witnessed by the magnetic susceptibility. The characteristic temperatures for the existing entanglement are determined by the entanglement witness. The experimental measurements of the magnetic susceptibility published previously are fitted by 
the model of one-dimensional Heisenberg mixed spin chain. The bound of negativity is obtained in the thermodynamical limit.

For an isotropic mixed spin $(S, 1 / 2)$ chain, the Hamiltonian $H$ can be expressed by

$$
\mathcal{H}=\sum_{i=1}^{n / 2} \mathcal{H}_{2 i}
$$

where $\mathcal{H}_{2 i}=J\left(\vec{S}_{2 i-1} \cdot \vec{s}_{2 i}+\vec{s}_{2 i} \cdot \vec{S}_{2 i+1}\right), \vec{S}$ and $\vec{s}$ are the spin vector of arbitrary spin $S$ and $1 / 2$ respectively. The number of spins is $n$, and the periodic condition of $n+1=1$ is assumed. The exchange interaction of $J>0$ and $J<0$ denote the antiferromagnetic and ferromagnetic case respectively. In the following discussion, an antiferromagnetic chain is considered.

The state at a thermal equilibrium temperature $T$ is $\rho(T)=e^{-H / k_{B} T} / Z$ where $Z$ is the partition function and $k_{B}$ is Boltzmann constant. The general expression for the magnetic susceptibility at zero-field can be written as

$$
\chi_{\alpha}=\chi=\frac{g^{2} \mu_{B}^{2}}{k_{B} T} \sum_{i, j=1}^{n} \mathcal{G}_{i j}^{\alpha},(\alpha=x, y, z)
$$

where $\chi$ is the magnetic susceptibility at the thermal equilibrium temperature $T, g$ is the g-factor, $\mu_{B}$ is the Bohr magneton, and $\mathcal{G}_{i j}^{\alpha}$ represents the correlation between the site $i$ spin and $j$ spin along $\alpha$ direction. It is assumed that the correlations of two spins which are not nearest neighboring are small enough to be ignored. This implies that the magnetic susceptibility can be approximately obtained by $\chi \simeq \frac{g^{2} \mu_{B}^{2} n}{k_{B} T}\left[\frac{1}{8}+\frac{S^{2}}{2}+\frac{\mathcal{G}_{1}}{3}\right]$, where $\mathcal{G}_{1}$ is the correlation between two nearest neighboring spins. In the isotropic mixed spin chains, any two nearest-neighboring thermal state is $\mathrm{SU}(2)$-invariant [26]. If the separability principle [11] is followed, $\mathcal{G}_{1}<-\frac{S}{2}$ will be satisfied for an entangled thermal state. In the thermodynamical limit, the entanglement witness $\mathcal{W}$ can be given by

$$
\mathcal{W}=\chi-\frac{g^{2} \mu_{B}^{2} n\left(12 S^{2}-4 S+3\right)}{24 k_{B} T}
$$

If the value $\mathcal{W}$ of the witness is negative, the thermal state is entangled. The separable state is for the value of $\mathcal{W}>0$. Then Eq.(3) is the generalized form of the entanglement 
Table 1: The characteristic temperatures for compounds*.

\begin{tabular}{cccc} 
compounds & $J\left(\mathrm{~cm}^{-1}\right)$ & spin $(S, 1 / 2)$ & approx. $T_{c}(K)$ \\
\hline $\mathrm{CN}$ & $5.12 k_{B}$ & $(1 / 2,1 / 2)$ & 4.7 \\
$\mathrm{ACu}(\mathrm{A}=\mathrm{Ni})$ & 81.4 & $(1,1 / 2)$ & 125 \\
$\mathrm{ACu}(\mathrm{A}=\mathrm{Co})$ & 18 & $(3 / 2,1 / 2)$ & 26 \\
$\mathrm{ACu}(\mathrm{A}=\mathrm{Fe})$ & 20 & $(2,1 / 2)$ & 32 \\
$\mathrm{ACu}(\mathrm{A}=\mathrm{Mn})$ & 23.44 & $(5 / 2,1 / 2)$ & 40 \\
* The data of $\mathrm{CN}$ are from $[20]$ and others are from $[25]$.
\end{tabular}

witness for the one-dimensional mixed spin $(S, 1 / 2)$ chain. From Eq.(3), the presence of the entanglement will be detected if the macroscopic susceptibility is measured and the parameters of the structure, i. e. the values of $g$ and $n$, are known. There is a characteristic temperature $T_{c}$ for $\mathcal{W}=0$. Below the temperature, an entangled thermal state is attained in the spin system. The characteristic temperature $T_{c}$ is plotted as a function of the mixed spin length $S$ and interaction $J$ in Fig.1. Here the height of the bar denotes the value of $T_{c}$. From Fig.1, it is seen that the values of $T_{c}$ are increased with the increase of the exchange interaction $J$. It is also clear that $T_{c}$ is almost linearly increased with the spin $S$ for the same interaction. By the numerical calculation, the characteristic temperature is approximately expressed as $T_{c} \approx J\left(a_{0} S+b_{0}\right) / k_{B}$ where $a_{0}$ and $b_{0}$ are two constants determined by the property of the material. The values of $T_{c}$ can be improved in the condition of high mixed spin and the strong exchange interaction. For an inorganic compound, the bimetallic chain $\mathrm{ACu}[25],(\mathrm{A}=\mathrm{Mn}, \mathrm{Fe}, \mathrm{Co}, \mathrm{Ni})$ has been synthesized. At a finite temperature, the macroscopic properties can be explained by the one-dimensional mixed spin chain. Using Eq.(3) and the measurements of the susceptibility [20, 25], the characteristic temperature $T_{c}$ for the entanglement is approximately given in Table 1. The special compound of $\mathrm{CN}(1 / 2,1 / 2)$ is also evaluated. The value of $T_{c}$ is about $4.7 \mathrm{~K}$ which is in good agreement with that given by [20]. The $\mathrm{ACu}$ compound corresponds to the Heisenberg spin $\left(S^{A}, 1 / 2\right)$ chain with $S^{A}=5 / 2,2,3 / 2,1$. The presence of the 
macroscopic entanglement is qualitatively described by the entanglement witness.

Although the entanglement witness $\mathcal{W}$ indicates the presence of the entanglement, it is still necessary to quantitatively study the relation of the quantum entanglement and the macroscopic thermodynamical observable. From the separability principle, the negativity [5] for the entanglement measurement can be introduced by

$$
\mathcal{N}(\rho)=\left|\sum_{i} \tau_{i}\right|
$$

where $\tau_{i}$ is the $i$ th negative eigenvalue of $\rho^{T}$ which is the partial transpose of the mixed state $\rho$. The measure corresponds to the absolute value of the sum of negative eigenvalues of $\rho^{T}$. The state $\rho$ is unentangled if all eigenvalues of $\rho^{T}$ are nonnegative. For an isotropic mixed spin $(S, 1 / 2)$ chain, thermal states between any two nearest neighboring spins $\rho_{2 i}$ is $\mathrm{SU}(2)$-invariant. The possible negative eigenvalue of $\rho_{2 i}^{T}$ can be written as

$$
\tau=\frac{S+2 \mathcal{G}_{1}}{\mathcal{D}(\mathcal{D}-1)}
$$

where the degree of degeneracy is $2 S$, and $\mathcal{D}=2 S+1$ represents the dimension of the mixed spin $\vec{S}$. Considering the effect of the correlation between any two spins, the lower bound of the negativity is obtained

$$
\mathcal{N}(\rho)>-\frac{6 \mathcal{W} k_{B} T}{\mathcal{D} g^{2} \mu_{B}^{2} n}
$$

where $\mathcal{N}(\rho)$ denotes the entanglement between two nearest neighboring spins in the thermodynamical limit. From Eq. (6), it is seen that the entanglement exists if Eq.(6) holds with $\mathcal{N}(\rho)>0$. The Eq.(6) is generalized to the isotropic mixed spin chain with the arbitrary spin of $S$. It is seen that the bound of negativity is proportional to the value of $-\mathcal{W}$. Meanwhile, the entanglement is decreased with the increase of the macroscopic value $\chi T$. The entanglement can be quantitatively evaluated in some degree when the magnetic susceptibility is measured. The bound of the entanglement may be decided by the measurements of the magnetic susceptibility. It is seen that the entanglement $\mathcal{N}(\rho)$ can affect the macroscopic magnetic properties. 
For the special case of $S=1 / 2$, the copper nitrate Cu-HTS [25] is approximately regarded as a one-dimensional antiferromagnetic Heisenberg chain. According to the experimental measurements of the magnetic susceptibility [24], the measured data of $\chi$ are shown by the circles in Fig. 2(a). When $T \rightarrow 0$, the magnetic susceptibility is finite. With the increase of the temperature, the values of $\chi$ is increased to a maximum at about $T=20 \mathrm{~K}$. Then the magnetic susceptibility declines as the temperature $T$ is increased. In Fig. 2(a), the experimental data are fitted by the theoretical curve when the interaction of $J=10.2 \mathrm{~cm}^{-1}$ and the g-factor of $g=2.06$. The solid line is the theoretical curve of the Heisenberg linear chain. The theoretical curve is in good agreement with the measured magnetic susceptibility. Above the temperature of about $T=12 K$, the effects of the correlation between non-nearest-neighboring spins can be reasonably negligible. This is the reason that the effects between non-nearestneighboring spins exponentially decrease as the separation of the sites is increased. However, at the low temperatures, these effects need to be considered. Using Eq.(6) and the measurements of the susceptibility, the experimental data of the bound of negativity is plotted by the circles in Fig. 2(b). The entanglement drops rapidly with the temperature, and vanishes at about $T=33 \mathrm{~K}$. For a pair of spins, the correlation function is theoretically expressed as $\mathcal{G}_{1}=-3\left(1-e^{-J / k_{B} T}\right) / 4\left(1+3 e^{-J / k_{B} T}\right)$. According to Eq.(4), the theoretical prediction of the negativity is plotted by the solid line in Fig. 2(b). Below $T=12 K$, the theoretical value is slightly higher. It is obvious that the theoretical curve obtained from Eq.(6) is in good agreement with the measurement of the susceptibility. To some extent, the quantum entanglement is quantitatively evaluated in the real solids.

The general case of the mixed spin with $S \neq \frac{1}{2}$ needs also to be investigated. As an example, the bimetallic compound chain of $\mathrm{NiCu}$ [25] can be considered as the mixed spin $(1,1 / 2)$ model. Above a certain temperature, the magnetic properties can be explained by the one-dimensional mixed spin chain. Due to the effects of three-dimensional and the long range exchange interaction, the magnetic susceptibility cannot be fitted using the one-dimensional linear chain at low temperatures. Above 
a certain temperature, the spin model can be simplified as the one-dimensional chain with the nearest neighboring interaction. Therefore, the macroscopic quantity of $\chi T$ and the theoretical prediction are shown in Fig. 3(a) at the temperature range of $25-250 K$. The circles denote the experimental data of $\chi T[25]$. Above the temperature of $T=8 K, \chi T$ is declined to the minimum at about $T=75 K$. The solid line is the theoretical fit by the one-dimensional mixed spin chain. It is seen that the values of $\chi T$ is in good agreement with the experimental data above $T=25 K$. Similarly, the bound of the negativity is illustrated in Fig. 3(b). The results of the experimental data are shown by the circles. From the measured data in Fig. 3(b), it is clear that the entanglement monotonously declines with the increase of the temperature. It is noted that the correlations of the non-nearest-neighboring spins will decrease with the temperature. Above the temperature $T=80 K$, the effect between two nearest neighboring spins $(1,1 / 2)$ dominates the macroscopic susceptibility. But the contributions from non-nearest-neighboring spins, i. e. $(1,1)$ and $(1 / 2,1 / 2)$, need still to be considered at the range of $T<75 K$. Thus, the correction item needs to be added to Eq.(6). By the numerical calculation, the additional item is proportional to $\mathcal{P}\left\langle\vec{S}_{2 i-1} \cdot \vec{s}_{2 i}\right\rangle$, where $\mathcal{P}$ is the polynomial decreasing function of the temperature. For the case of $\mathrm{NiCu}$, the function can be expressed as $\mathcal{P} \sim 0.11\left(J / k_{B} T\right)-0.07\left(J / k_{B} T\right)^{2}$. Above the temperature $T=122 k$, there is no entanglement in the compound. For low temperatures of $T<25 K$, the spin model cannot be regarded as a one-dimensional linear chain with the antiferromagnetic interaction. Therefore, only the entanglement above a certain temperature can be calculated by Eq.(6). For a pair of spins, the correlation is given by $\mathcal{G}_{1}=-5\left(1-e^{-3 J / 2 k_{B} T}\right) /\left[6\left(1+2 e^{-3 J / 2 k_{B} T}\right)\right]$. From Eq.(4), the theoretical value of the negativity is plotted by the solid line. It is seen that the theoretical curve is slightly higher than the results of the susceptibility measurements when the temperature is low.

From the two kinds of solid compounds, it is demonstrated that the quantum entanglement plays an essential role in the macroscopic phenomena. The magnetic susceptibility embodies the quantum entanglement. From the experimental measurements of 
the susceptibility, the entanglement is quantitatively evaluated. It is likely that the quantum entanglement can be used as a resource of the quantum information processing in the thermodynamical limit.

In summary, the entanglement in a general mixed spin $(S, 1 / 2)$ chain is investigated using the macroscopic magnetic susceptibility. The entanglement witness for arbitrary spin $S$ is expressed by the susceptibility. The entanglement witness determines a characteristic temperature $T_{c}$ below which an entangled thermal state can be obtained. It is seen that the values of $T_{c}$ are nearly proportional to the exchange interaction $J$ and the mixed spin $S$. The characteristic temperatures for the $\mathrm{ACu}$ compounds are approximately determined. It is likely that the entanglement can be detected at a rather high temperature in the solids with high spin and the strong interaction. The relation of the quantum entanglement and the macroscopic susceptibility is deduced. The bound of negativity can be obtained by the experimental measurements of the susceptibility. To some extent, the entanglement can be quantitatively evaluated by the susceptibility. It is demonstrated that the macroscopic magnetic properties can be affected by the quantum entanglement in the real solids.

It is a pleasure to thank Yinsheng Ling, Jianxing Fang, and Qing Jiang for their many fruitful discussions about the topic. 


\section{Reference}

1. D. P. DiVincenzo and D. Eacon and J. Kempe and G. Burkard and K. B. Whaley, Nature(London)408, 339(2000).

2. A. Vaziri and G. Weihs and A. Zeilinger, Phys. Rev. Lett.89, 240401(2002).

3. W. K. Wootters, Phys. Rev. Lett.80, 2245(1998).

4. V. Vedral and M. B. Plenio, Phys. Rev. A57, 1619(1998).

5. G. Vidal and R. F. Werner, Phys. Rev. A65, 032314(2002).

6. M. C. Arnesen and S. Bose and V. Vedral, Phys. Rev. Lett.87, 017901(2001).

7. G. L. Kamta and A. F. Starace, Phys. Rev. Lett.88, 107901(2002).

8. S. Gu and S. Deng and Y. Li and H. Lin, Phys. Rev. Lett.93, 086402(2004).

9. C. Lunkes and C. Brukner and V. Vedral, Phys. Rev. Lett.95, 030503(2005).

10. S. Ghosh and T. F. Rosenbaum and G. Aeppli and S. Coppersmith, Nature(London)425, 48(2003).

11. A. Peres, Phys. Rev. Lett.77, 1413(1996).

12. X. Wang and P. Zanardi, Phys. Lett. A301, 1(2002).

13. M. R. Dowling and A. C. Doherty and S. D. Bartlett, Phys. Rev. A70, 062113(2004).

14. K. Maruyama and F. Morikoshi and V. Vedral, Phys. Rev. A71, 012108(2005).

15. G. Toth, Phys. Rev. A71, 010301(R)(2005).

16. P. Hyllus and O. Guhne and D. Bruss and M. Lewenstein, Phys. Rev. A72, 012321(2005).

17. M. Lewenstein and B. Kraus and J. I. Cirac and P. Horodecki, Phys. Rev. A62, 052310(2000).

18. A. Sanpera and D. Bruss and M. Lewenstein, Phys. Rev. A63, 050301(R)(2001).

19. M. Wiesniak and V. Vedral and C. Brukner, e-print quant-ph/0508193.

20. C. Brukner and V. Vedral, and A. Zeilinger, e-print quant-ph/0410138.

21. M. Wiesniak and V. Vedral and C. Brukner, e-print quant-ph/0503037.

22. T. Vertesi and E. Bene, e-print quant-ph/0503726.

23. Q. Jiang and Z. Y. Li, Phys. Rev. B40, 11264(1989). 
24. W. E. Hatfield, J. Appl. Phys.52, 1985(1981).

25. P. J. van Koningsbruggen and O. Kahn and K. Nakatani and Y. Pei and J. P. Renard and M. Drillon and P. Legoll, Inorg. Chem.29, 3325(1990).

26. J. Schliemann, Phys. Rev. A68, 012309(2003). 


\section{Figure Captions}

Fig. 1 .

The characteristic temperature $T_{c}$ is plotted when the mixed spin $S$ and the exchange interaction $J$ are varied. The height of the bar denotes the value of $T_{c}$. It is linearly increased with the increasing values of $S$ and $J$.

\section{Fig. 2 .}

(a). The magnetic susceptibility of the compound $\mathrm{Cu}-\mathrm{HTS}$ is plotted as a function

of the temperature $T$. The circles are the measured data [24]. The parameters are taken from Ref. [24] with the interaction $J=10.2 \mathrm{~cm}^{-1}$ and the g-factor $g=2.06$. The theoretical prediction is plotted by the solid line.

(b). Based on the experimental measurements, the bound of the negativity is calculated and represented by the circles. The solid line is the theoretical curve.

\section{Fig. 3.}

(a). The measurements of the magnetic susceptibility for $N i C u$ from Ref. [25] are denoted by the circles. Above the temperature $T=75 K$, the magnetic properties can be explained by the one-dimensional mixed spin chain. The solid line is the theoretical fit with the interaction $J=81.4 \mathrm{~cm}^{-1}$ and the g-factor $g=2.15$.

(b). From the experimental data of $\chi T$, the bound of the negativity is plotted as the circles. The solid line is the theoretical fit. 


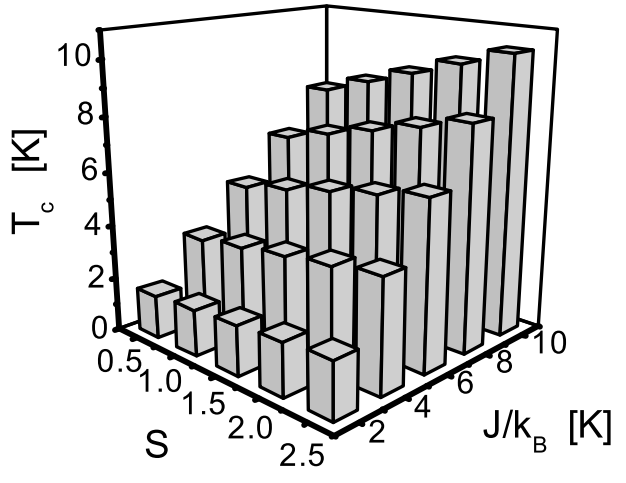



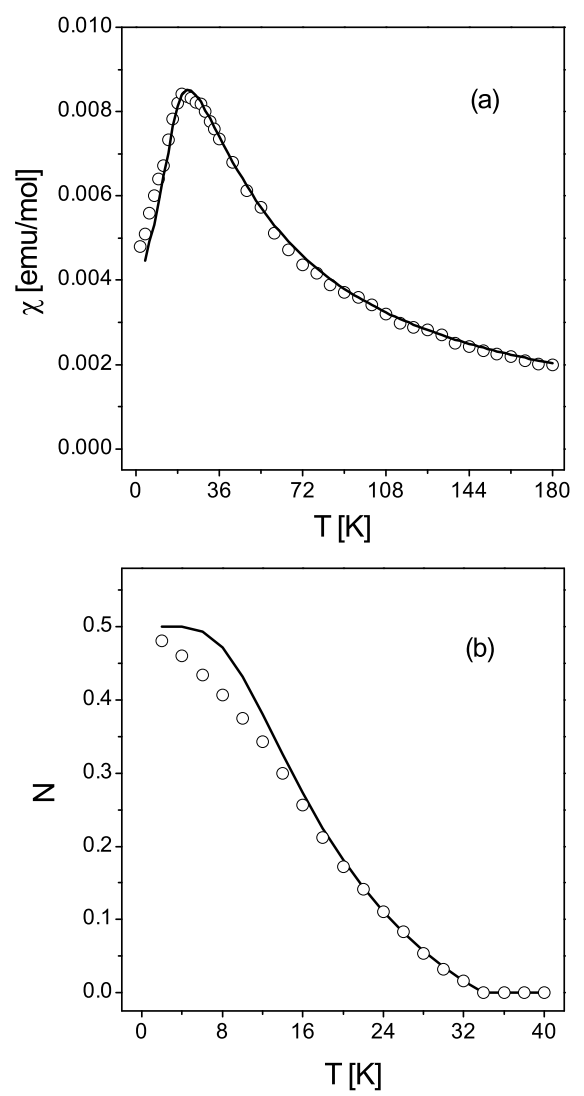

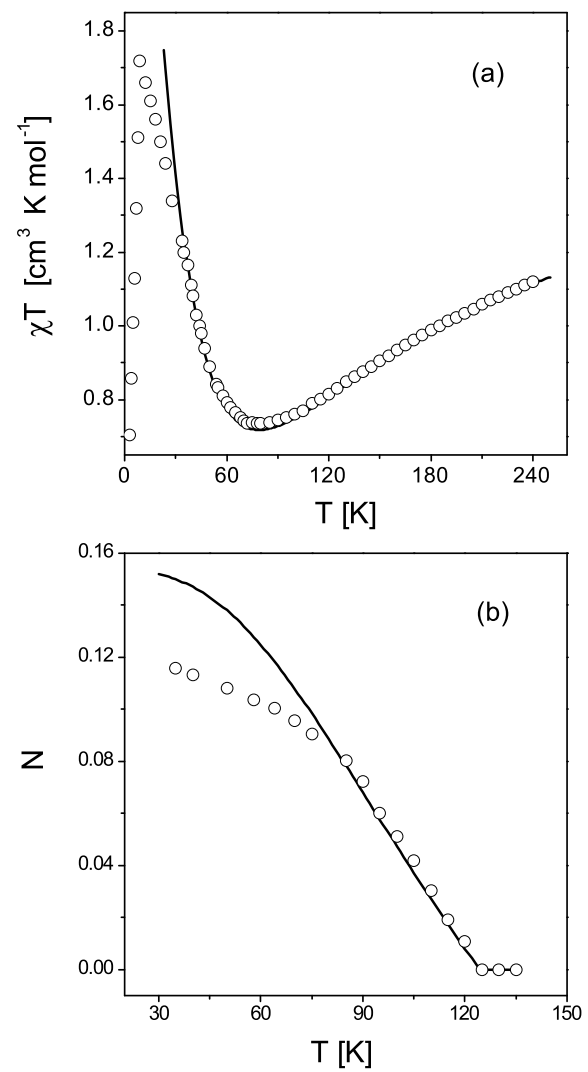